\title{
Adult Presentation of a Complete Second Branchial Cleft Fistula Diagnosed by US and CT, Autosomal Dominant Transmission in Three Members of the Family: Case Report
}

\author{
Patrick Mailleux, Yorick Lismonde \\ Department of Imaging, Clinique St Luc, Bouge, Belgium \\ Email: p.mailleux@gmail.com
}

How to cite this paper: Mailleux, P. and Lismonde, Y. (2020) Adult Presentation of a Complete Second Branchial Cleft Fistula Diagnosed by US and CT, Autosomal Dominant Transmission in Three Members of the Family: Case Report. Open Journal of Medical Imaging, 10, 125-131. https://doi.org/10.4236/ojmi.2020.102012

Received: May 15, 2020

Accepted: June 13, 2020

Published: June 16, 2020

Copyright ( 2020 by author(s) and Scientific Research Publishing Inc. This work is licensed under the Creative Commons Attribution International License (CC BY 4.0).

http://creativecommons.org/licenses/by/4.0/

\begin{abstract}
Branchial arch anomalies can arise from the four first branchial arches, but the most encountered cases are from the second one. Second branchial arch cysts and abscesses occur mainly in older children or young adults while fistulae are discovered in young children. We report a case of complete second branchial arch fistula of Bailey III type with adult complaints of painful swelling and local reddishness followed by spontaneous discharge and disappearance of complaints. Diagnosis was based on ultrasound and confirmed by CT scan, with the classic "beak sign" visible on both exams. Three cases were encountered in the family, with no otologic or kidney symptoms, which is quite different from the classical branchiootorenal syndrome which associates severe inner ear and kidney congenital anomalies.
\end{abstract}

\section{Keywords}

Branchial Cyst, Branchial Arc, Fistula, CT Scanner, Ultrasound

\section{Introduction}

Congenital anomalies are often encountered in the pediatric population and always stay on top of the gamut of differential diagnoses. But some congenital anomalies may be sparsely symptomatic during childhood and have late more explosive symptoms. In the case of second branchial arch anomalies, fistulae, cysts and abscesses can be encountered: fistulae in young children, cysts and abscesses in older children and young adults. We present here a case of a 60 -year-old lady presenting at the emergency department with symptoms caused by an inflamed and temporarily obstructed second branchial arc fistula. Ultra- 
sound and CT both allowed precise diagnosis. A discussion follows about the genetic transmission of this pathology in the family.

\section{Case Report}

A 60 years old female patient complained of left anterolateral neck swelling at the level of the thyroid gland. The area was reddish and painful. Fear of infection led her general practitioner (GP) to prescribe for her oral antiobiotics (amoxicilline and clavulac acid Clavucid), a treatment she took for four days without improvement. Her GP then sent her to the emergency department for imaging and adjustment of medical treatment if needed. There, the painful left anterolateral neck swelling above the level of the parotid gland was still present, the patient had no fever, and the laboratory investigations showed C Reactive protein level to be in the normal range $(4.18 \mathrm{mg} / \mathrm{l})$, with slightly elevated white blood cell count $\left(10,300 / \mathrm{mm}^{3}\right)$ without an increase in neutrophils $(61 \%)$.

Ultrasound showed that the swelling was caused by a fluid-filled tubular structure extending longitudinally in the neck, lateral to the thyroid gland and anteromedial to the sternocleidomastoid (SCM) muscle, anterior to the common carotid. The distal part of the collection had $10 \mathrm{~mm}$ of diameter, with a $2-3 \mathrm{~mm}$ thick wall, and extended as a kind of fibrous tract through the subcutaneous space to a small skin lesion at the anterior border of the SCM (Figure 1(A)). Higher up, the fluid component was not as large ( $5 \mathrm{~mm}$ of diameter) but the structure had thicker $4 \mathrm{~mm}$ wall presenting some hypervascularity on doppler imaging (Figure 1(B)). The higher third of the tubular structure did not contain fluid and could be followed between the internal carotid and the branches of the external carotid, bowing medially below the level of a normal submandibular gland, forming the classical "beak" sign, pathognomonic of the pathology [1] [2] (Figure 1(B)). When specifically asked, the patient told that the small punctum at the anterior border of the SCM (Figure 2) exists since childhood, sometimes as a kind of fistula, with intermittent purulent discharge since her childhood, but that it became unproductive 10 years ago.

A CT scan with intravenous contrast injection was performed one day later but during the night, the cutaneous orifice had reopened with spontaneous discharge of green pus. CT exam confirmed all the ultrasound findings but the cystic area was not fluid-filled and dilated anymore. The CT exam clearly demonstrates that the "fistula" has no relation with the piriform sinus, it can be followed between the branches of internal and external carotid to the lateral wall of the oropharynx (Figure 3 and Figure 4).

The medical management of the patient was simple: neck swelling, pain and local reddishness disappeared following the spontaneous discharge. So that the emergency physician told the patient to take the antibiotic treatment for a few more days and to consult an ENT (ear-nose-throat) specialist. As all symptoms had completely vanished without any residual drainage, the ENT consultation did not take place. Eight years later, the patient is fit and well without any recurrence of symptoms. 


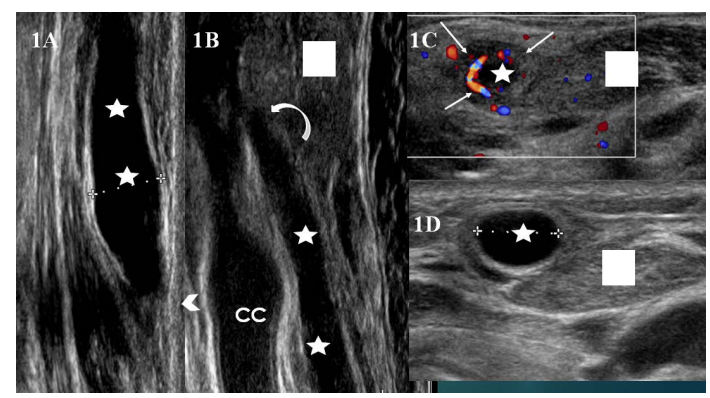

Figure 1. (A) Sagittal left paramedial ultrasound view: cystic dilation (star) just above the cutanaeous anomaly (symbolized by an arrowhead). (B) Coronal ultrasound showing the fistulous tract in the upper neck (star) "beaking" (curved arrow) below the submandibular gland (square). Common carotid: CC. (C) Axial slice through the upper part of fistula tract (star), poorly filled, below the submandibular gland. Doppler colour signal in the thickened wall (arrows), sternocleidomastoid (square). (D) Axial through the dilated inferior part of the tract (star), sternocleidomastoid (square).

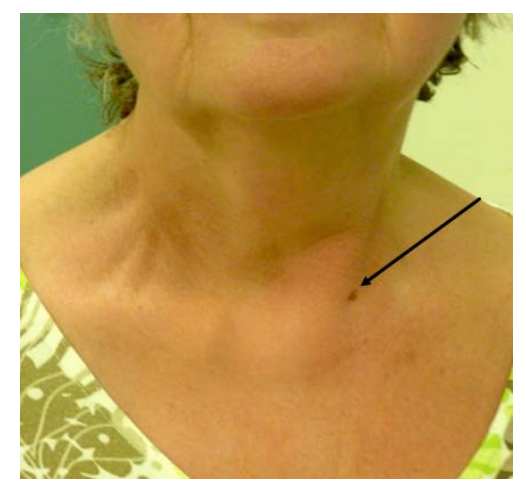

Figure 2. Day 2. The swelling disappeared, the tiny punctum (black arrow) at the anterior border of the base of the SCM muscle is visible. It is present since infancy, with intermittent mucoid or purulent discharge.

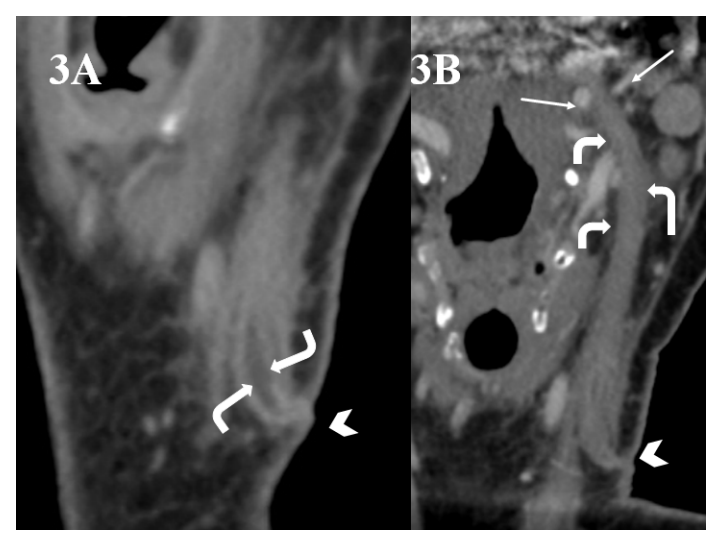

Figure 3. (A) CT scan with intravenous contrast: oblique coronal reconstruction showing the inferior part of the fistula tract leading to the cutaneous opening of the fistula at the skin. The lower part of the tract still contains some fluid (curved arrows), crosses the subcutaneous space to the skin opening (arrowhead). (B) oblique coronal reconstruction: the upper part of the fistula tract (curved arrow) is seen going between the external and internal carotid arteries (arrows), plunging medially to the oropharyngeal wall at the level of the tonsils. 


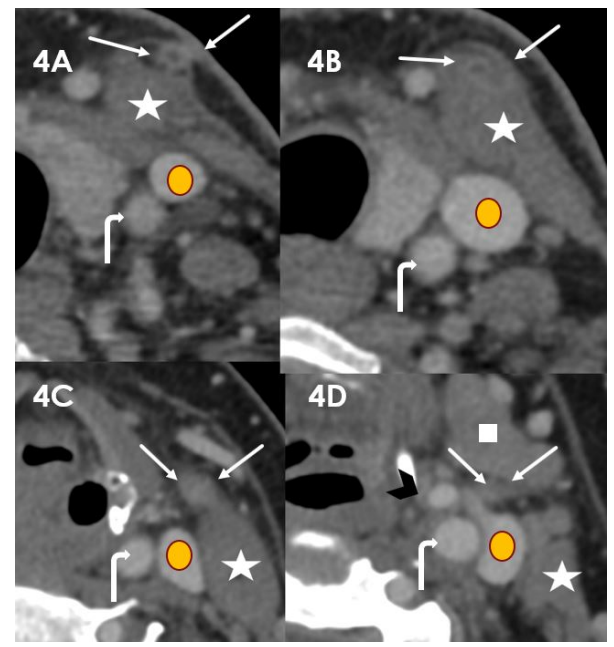

Figure 4. Axial CT slices with contrast injection, from the level of the external skin orifice below (A) to the level of submandibular gland above (D). Fistulous tract (thin arrows), SCM (star), jugular vein (circle), common carotid and internal carotid (curved arrow), external carotid (arrowhead), submandibular gland (square).

The family history relates the same findings, always unilateral in two other patients: one in a male child of the patient, on the right side, and one in a male grand-child of another of her boys, on the left side. Mother, son and grand-son all had only mild symptoms with intermittent discharge, often related to upper airways infection during childhood, without abscess formation or general septic symptoms. Only the grand-child was operated upon, mainly because he also had to have adenoidectomy. None of the three patients had otologic problems or kidney anomalies.

\section{Discussion}

Branchial cleft cysts are congenital anomalies arising from the first through fourth branchial clefts [1] [2] [3] [4] due to incomplete regression of branchial cleft structures. Anomalies are described in the four first branchial arches: cysts and fistulae of the first arch are centered on the ear, the parotid gland and the facial nerve, they are most commonly seen in middle-aged women with a history of recurrent parotid abscess unresponsive to treatment and drainage [1]. Fistulae of the third and fourth arch are difficult to differentiate from each other and present as lower cervical suppuration and mimic relapsing suppurative thyroiditis, with only a few reported cases, in children and young adults [1].

The most common type of branchial cleft lesions arises from the second cleft in $40 \%$ to $95 \%$ of the cases [3]. Those second branchial cleft anomalies can present in one of three forms: cysts, sinuses or fistulae. The course of the second branchial cleft starts from an external opening on the neck skin anterior and medial to the SCM, travels up deep to the platysma, beaks medially between the internal and external carotid arteries to reach the tonsillar fossa. Thus, cyst or sinuses of the second cleft can be found anywhere along this course. If the fistula 
is complete, it drains itself from time to time through a sinus opening, while the more frequent incomplete fistula will present as a simple cystic structure in the subcutaneous tissue of the area. Those cysts have an epithelial lining without external openings, and as such may be asymptomatic or be noticed accidentally in adult patients. They can become symptomatic with some swelling or regression, and common secondary infection.

In the adult population, the branchial cyst is a frequent differential diagnosis problem in patients presenting with cervical cystic or cystic-looking masses. Those congenital anomalies present usually in early adult age (10 to 40 years) [1], sometimes after a small trauma of infection. They are usually located just under the angle of the mandible, anterior to the SCM. In adult patients, the main diagnostic consideration is whether the cystic lesion represents a metastatic lymph node and subsequent imaging is directed at identifying a primary neoplastic lesion. This is particularly true if there is no history of chronic neck fullness and no history of a recurrent mass following upper respiratory tract infections [5].

On the other hand, sinus tracts and complete fistulae are encountered mainly in a pediatric population. Sinus tracts may communicate either externally with skin as a visible punctum, or internally with the pharynx or larynx, to be seen only on endoscopy or fistulography [3]. Branchial cleft fistulae are true communications connecting the pharynx with the external skin, they are rare in the adult population [6].

Bailey's classification divided the second branchial arch anomalies in 4 groups [7]. Type I is the most superficial type, directly under the superficial cervical fascia, anterior to the sternocleidomastoid muscle. Type II, the more frequent one lies, under the middle cervical aponeurosis, anterior and lateral to the carotid vessels. Type III extends to the pharynx, between internal and external carotid artery while the very rare type IV is located lateral to the pharyngeal wall and medial to the carotid artery.

Our patient had a Bailey III fistula with distal punctum and intermittent discharge. Both US and CT allowed a complete workup of the anomaly, from the tonsils, going laterally between internal and external carotid arteries, then along the SCM to end at the level of the skin above the collarbone. In our patient, on US (Figure 1) and CT (Figure 3 and Figure 4), the "beak sign" was clearly seen, the upper part of the fistulous tract pointing medially between the internal and external carotid arteries. That sign is considered to be a pathognomic imaging feature of a second branchial fistula, specifically a Bailey type III [1] [2]. The usual treatment in the pediatric population is complete resection of the fistula tract, with few recurrences [7]. Due to the sparse symptoms in the patient and her son, surgery was never considered.

Hereditary transmissible branchial tract anomalies were described [8] [9], the more common syndrome is the "branchiootorenal syndrome" (BOR syndrome) associating severe congenital deafness, branchial cleft sinuses, cervical fistulas and renal anomalies, which range from mild hypoplasia to bilateral renal agene- 
sis. None of the three patients in the family of this case had otologic problems or kidney anomalies, so that the classical genetic branchiootorenal syndrome seems to be excluded. It could be another entity, or a variation with very partial penetrance.

\section{Conclusion}

In the adult population, the more frequently encountered second branchial cyst anomalies are the branchial cysts, symptomatic when increasing or infected. This 60-year-old patient presented with painful neck swelling because of inflammation and transient filling of a complete Bailey III fistula. Its later opening leads to a spontaneous emptying of the cyst and resolution of her symptoms with no later recurrence after 8 years. Ultrasound and CT correctly analyzed the lesion. Similar cases of unilateral fistulae with no otologic or kidney symptoms were present in the family, with a dominant autosomal transmission pattern.

\section{Declaration}

Informed consent was obtained from the patient.

\section{Conflicts of Interest}

The authors declare no conflicts of interest regarding the publication of this paper.

\section{References}

[1] Koeller, K.K., Alamo, L., Adair, C.F. and Smirniotopoulos, J.G. (1999) Congenital Cystic Masses of the Neck: Radiologic-Pathologic Correlation. RadioGraphics, 19, 121-153. https://doi.org/10.1148/radiographics.19.1.g99ja06121

[2] Harnsberger, H. (1995) Handbook of Head and Neck Imaging. 2nd Edition, Mosby-Yearbook, St Louis.

[3] Coste, A.H., Lofgren. D.H. and Shermetaro, C. (2020) Branchial Cleft Cyst. StatPearls Publishing, Treasure Island (FL). https://www.ncbi.nlm.nih.gov/books/NBK499914/

[4] Johnson, J.M., Moonis, G., Green, G.E., Carmody, R. and Burbank, H.N. (2011) Syndromes of the First and Second Branchial Arches, Part 2: Syndromes. American Journal of Neuroradiology, 32, 230-237. https://doi.org/10.3174/ajnr.A2073

[5] Adams, A., Mankad, K., Offiah, C., et al. (2016) Branchial Cleft Anomalies: A Pictorial Review of Embryological Development and Spectrum of Imaging Findings. Insights Imaging, 7, 69-76. https://doi.org/10.1007/s13244-015-0454-5

[6] Singh, A.K. and Asthana, A. (2013) The Complete Second Branchial Cleftfistula-A Case Report. Otolaryngology Online Journal, 3, No. 4.

https://www.alliedacademies.org/articles/the-complete-second-branchial-cleft-fistul a-a-case-report.pdf

[7] Shen, L.F., Zhou, S.H., Chen, Q.Q. and Yu, Q. (2018) Second Branchial Cleft Anomalies in Children: A Literature Review. Pediatric Surgery International, 34, 1251-1256. https://doi.org/10.1007/s00383-018-4348-8

[8] Amer, I., Falzon, A., Choudhury, N. and Ghufoor, K. (2012) Branchiootic Syn- 
drome-A Clinical Case Report and Review of the Literature. Journal of Pediatric Surgery, 47, 1604-1606. https://doi.org/10.1016/j.jpedsurg.2012.05.016

[9] Senel, E., Kocak, H., Akbiyik, F., Saylam, G., Gulleroglu, B.N. and Senel, S. (2009) From a Branchial Fistula to a Branchiootorenal Syndrome: A Case Report and Review of the Literature. Journal of Pediatric Surgery, 44, 623-625.

https://doi.org/10.1016/j.jpedsurg.2008.10.034 